\title{
Reduction of response latency in monkeys by a procedure of differential reinforcement'
}

JOSEF M. MILLER ${ }^{2}$, MITCHELL GLICKSTEIN AND WILLIAM C. STEBBINS ${ }^{2}$

UNIVERSITY OF WASHINGTON

The response latency of monkeys performing in a visual reaction time situation was reduced when a contingency for differential reinforcement of brief-latency responses (DRB) was introduced. Introduction of the DRB contingency was also associated with a reduction in the variability of response latency.

Animals may be trained to perform responses of brief latency to the onset of an exteroceptive stimulus in a reaction time (RT) situation (Stebbins \& Lanson, 1961; Stebbins \& Reynolds, 1964). The behavioral situation with animals is structured similarly to the classical human RT situation. Ss are trained to depress a key following the onset of a "ready signal," hold the key down during a variable "foreperiod," and to release the key following the onset of a second stimulus. The time interval between onset of the second stimulus and key release is the measured RT. Like the human RT measure, this measure of behavior in animals has been shown to be sensitive to changes in the intensity of stimulation (Stebbins \& Miller, 1964; Stebbins, 1966).

In the human RT experiment Ss are usually instructed to "respond as quickly as possible." Preliminary work (Stebbins \& Miller, 1964), has suggested that the addition of a contingency for differential reinforcement of brief latency responses (DRB) may yield shorter RTs and more stable performance than previously observed. This contingency could serve as a substitute for the verbal instructions used in the human RT situation. This paper is a report of the behavioral effects of instituting a DRB contingency on RT performance in monkeys.

\section{Method}

Three pigtail monkeys (Macaca nemestrina), ranging in weight from 3 to $8 \mathrm{~kg}$, were used as Ss: F, Z, and B. Throughout the experiment they were maintained in primate restraining chairs.

The Ss were trained in a darkened, audiometric testing chamber (IAC, model 400A) to await the onset of a clearly audible $1 \mathrm{kHz}$ tone, then to press a telegraph key (Foringer) and hold it down through a variable $0-5 \mathrm{sec}$. foreperiod, and, at the onset of a light, to release the key. The light was produced by an $\mathrm{Ne} 40$ bulb which was viewed through a $1 \mathrm{~cm}$ hole located $15 \mathrm{~cm}$ from the monkey's eyes. The intensity of the light measured independently by three observers with a Macbeth Illuminometer was approximately $8 \mathrm{ft}-\mathrm{c}$. Details of the training procedure have been reported previously (Stebbins \& Miller, 1964). Stimulus presentations were separated by a $35-\mathrm{sec}$. intertrial interval. Inappropriate key presses, prior to onset of the tone, or inappropriate releases, prior to onset of the light, resulted in the start of the 35-sec. intertrial interval. Key release in the presence of the light was followed by delivery of a $1-\mathrm{gm}$ whole-diet, bananaflavored, monkey pellet (Ciba). Monkey F's latencies were recorded on a Standard Electric Timer accurate to $0.01 \mathrm{sec}$. Response latencies of Monkeys $\mathrm{Z}$ and $\mathrm{B}$ were recorded using a solid-state digital clock, accurate to $1 \mathrm{msec}$.

The timer controlling the length of the DRB criterion interval was arranged in such a way that if key release did not occur within a preset period of time following onset of the light, the tone and light

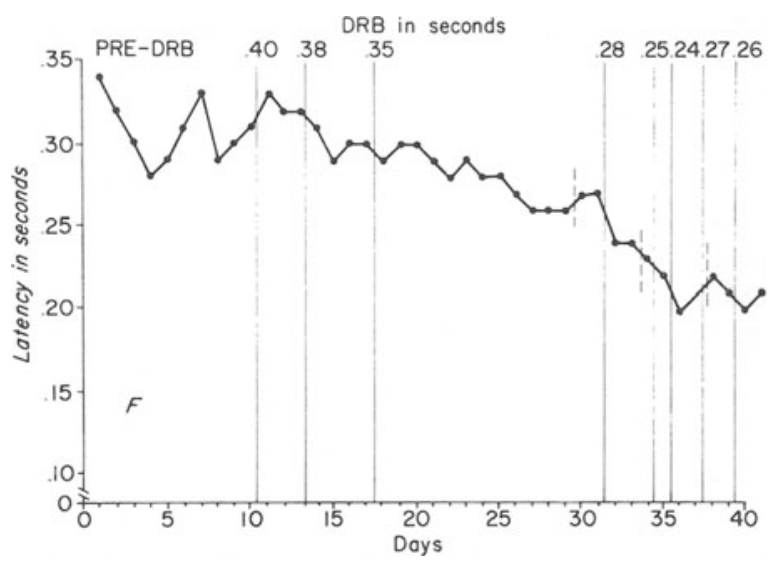

Fig. 1. Median daily reaction times of subject $F$ with decreasing DRB criterion interval. On day 37 performance was unstable.

terminated, and no reinforcement was delivered. The DRB contingency was introduced for Monkey $F$ after extensive behavioral training (over 2 years), for Monkey $\mathrm{Z}$ after one month of preliminary training, and for Monkey $\mathrm{B}$ the contingency was made an initial condition of the RT training procedure. The DRB criterion interval was decreased in successive steps with a decrease in response latency and stable per formance being the criteria for further reduction in the interval.

\section{Results}

Figure 1 illustrates the effects of instituting the DRB contingency on Monkey $F^{\prime}$ 's median response latency. With the introduction of DRB, F's latency showed a progressive decrease until the DRB criterion interval reached $0.25 \mathrm{sec}$. When the interval was lowered to 0.24 sec., the animal's behavior became unstable with increased variability and a greater number of inappropriate responses. Increasing the interval to $0.27 \mathrm{sec}$. re-established stable performance. F's initial median

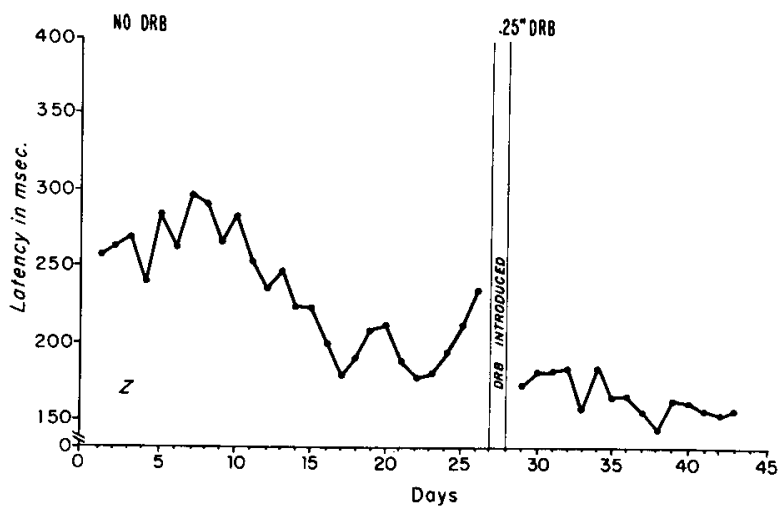

Fig. 2. Median daily latencies of subject $Z$ during first 43 days of RT training, before and after application of $0.25 \mathrm{sec}$. DRB contingency. 


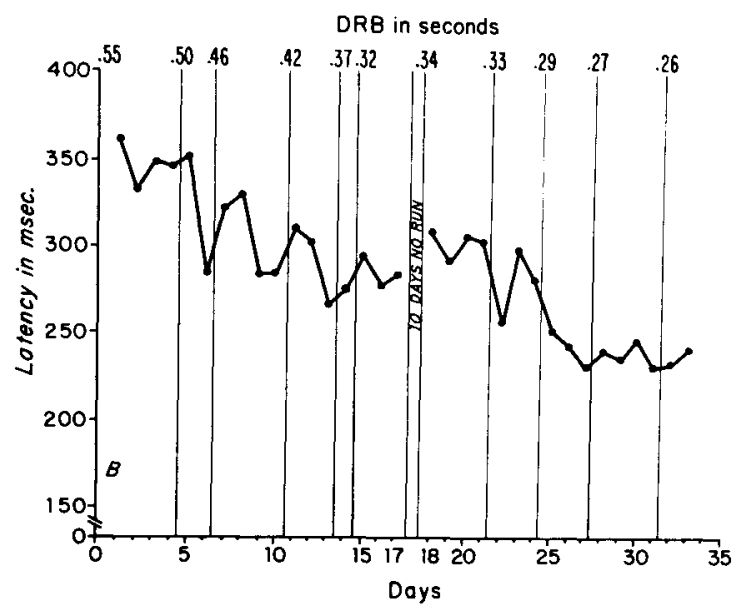

Fig. 3. Median daily latencies of subject $B$ during first 33 days of RT training, under decreasing DRB criterion interval.

latencies were about $0.32 \mathrm{sec}$; following DRB training they stabilized at approximately 0.21 sec. $^{3}$ Figure 2 shows response latencies during initial RT training and the effect of DRB on performance for Monkey $\mathrm{Z}$. The DRB criterion interval was introduced and then reduced as rapidly as possible on days 27 and 28 of $\mathrm{RT}$ training. Introduction of DRB reduced the response latencies to a low value, one which had been approached only sporadically under pre-DRB conditions. Median response latencies for Monkey $\mathrm{Z}$ were thereafter maintained at this low value. The performance of Monkey $B$, with whom the DRB contingency was made a part of the initial training procedures, is illustrated in Fig. 3. Median response latencies began to shorten immediately and reached a stable level in about 27 days of training. From day 27 to day 32 median daily latencies were between 230 and $250 \mathrm{msec}$.

Aside from the observed effects on median latency,

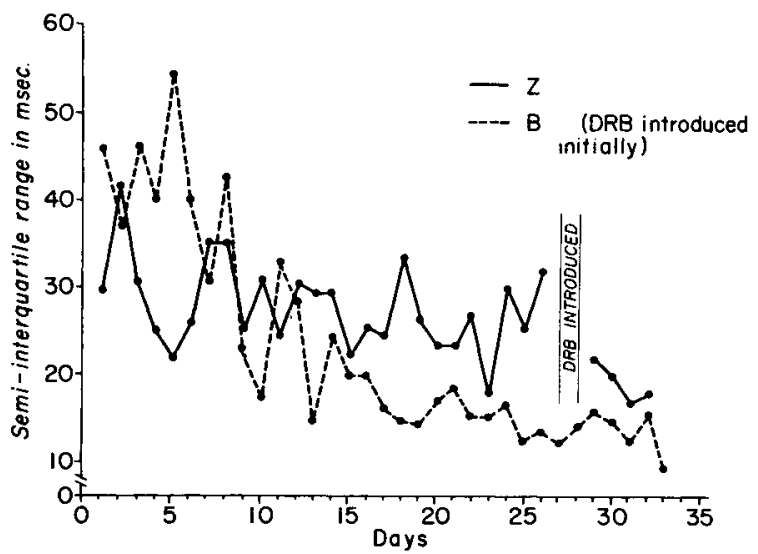

Fig. 4. Daily semi-interquartile range for subjects $Z$ and $B$ during first 33 days of RT training. the DRB contingency tended to decrease the variability of RTs. Prior to introduction of DRB, F's semiinterquartile ranges were between 0.02 and $0.03 \mathrm{sec}$. During the training period illustrated in Fig. 1, F's semi-interquartile ranges decreased to between 0.01 and $0.02 \mathrm{sec}$. Figure 4 illustrates a similar effect on the variability of performance of $Z$ and $B$. Little or no change occurred in variability of $Z$ 's performance until the DRB contingency was introduced, at which time the variability clearly decreased. B's response began to exhibit a decrease in variability following the fifth day of DRB training and stabilized at a semi-interquartile range of approximately $1 \pm \mathrm{msec}$.

\section{Discussion}

The decrease in latencies seen in F's performance clearly demonstrates that the DRB contingency is effective in shortening RTs. The continjency had a less striking effect on the performance of $\mathrm{Z}$. This animal's performance suggests that following initial training in the RT situation, response latencies may attain a low level and that they may then increase to some extent. For Monkey $Z$ the DRB contingency appears to reinstitute and maintain brief response latencies.

The observed decrease in variability of performance does not appear to be due only to a decrease in median response latency. $Z$ 's minimal median latencies before and immediately after introduction of DRB are equal; however, the variability is much reduced following introduction of the $0.25 \mathrm{sec}$. DRB contingency.

These findings have been corroborated by one of the authors (W.S.) with two cynomologous monkeys in an auditory RT situation. Thus the evidence indicates that as the DRB criterion interval is reduced, response latency decreases. Eventually a point is reached at which further reduction in the interval causes no further reduction in latency and/or the S's performance becomes unstable. In addition to producing short latency responses, the DRB contingency appears to decrease variability of latency. These effects of the DRB contingency are those expected of a contingency which forces the $S$ to "respond as quickly as possible."

\section{References}

Miller, J. M. Neural circuits and reaction time performance in monkeys. Ph.D. dissertation. 1965. Departments of Psychology, and Physiology and Biophysics, University of Washington, Seattle, Washington.

Miller, J. M., \& Glickstein, M. Reaction time to cortical stimulation. Science, 1964, 146, 1594-1596.

Stebbins, W. C. Auditory reaction time and the derivation of equal loudness contours for the monkey. J. exp. Anal. Behav., 1966, 9, 135-142.

Stebbins, W. C., \& Lanson, R. N. A technique for measuring the latency of a discriminative operant. J. exp. Anal. Behav., 1961, 4, 149-155.

Stebbins, W. C., \& Miller, J. M. Reaction time as a function of stimulus intensity for a monkey. J. exp. Anal. Behav., 1964, 7, 309-312.

Stebbins, W. C., \& Reynolds, R. W. Note on change in response latency following discrimination training in the monkey. $J$. exp. Anal. Behav., 1964, 7, 229-231.

\section{Notes}

1. The investigation was supported in part by U. S. Public Health Service Grants MH 06722, NB 16192-01, GM 666-05.

2. Now at Kresge Hearing Research Institute, University of Michigan Medical School, Ann Arbor, Michigan.

3. This work was done as part of a larger investigation on the neural circuits involved in reaction time performance (Miller, 1965; Miller \& Glickstein, 1964). In Fig, 1, at each of the times indicated by the dashed lines a few days of training and testing were interspersed in which light stimulus was replaced by direct electrical stimulation of the visual cortex. 